\title{
Research on Modularization and Association of Elderly Care Services in Big Data Environment
}

\author{
Yan $\mathrm{Zhao}^{1, *}$ \\ ${ }^{1}$ school of economics and management, Inner Mongolia normal university, Hohhot 010022, China
}

\begin{abstract}
At this stage, China is in the stage of building a well-off society in an all-round way, and the application of big data has positive significance for the grasp of China's national conditions and the laws of scientific development. At the same time, big data applications can also provide support for the development of the elderly care service industry. This article summarizes the modular content of elderly care services based on previous work experience. The author discusses the data association analysis of distributed elderly care services in a big data environment from four aspects: the construction of distributed association classifiers, the incremental mining of association classifiers based on constraints, the running process of the distributed association classifier model, and the evaluation of actual elderly care services.
\end{abstract}

\section{Introduction}

Under the influence of big data technology, there are obvious innovations and changes in pension services. Relevant data show that in 2020, the proportion of people over 60 years old in China will reach $17.8 \%$. Facing such a huge group of elderly people, we need to rely on big data technology to ensure the interconnection and intelligence of the elderly care service model. We should establish precise elderly care services, which is also the specific requirement for elderly care work in China at this stage. Therefore, the relevant departments need to establish a mining model with high satisfaction with the help of the association classification method, so as to accurately match the demand side and the supply side of elderly care services. We should clarify the corresponding constraints to ensure that the final mining results are more effective.

\section{The Organic Integration of Big Data and The Elderly Service Industry}

With the continuous development of network technology, the amount of data stored by people has increased significantly. This has prompted the data to complete the transformation from quantitative change to qualitative change, which is also the specific manifestation of "big data". Throughout the entire data science research process, massive data is the main research object, and it uses data mining and other methods to clarify the laws that exist in the data. From the perspective of supporting dimensions, big data is a technology platform. In the process of work, the collection and storage of actual data are different from conventional data, and their development requires software and hardware technical support. If it is analyzed from the perspective of tools, big data is a good research tool. At this stage, big data has been applied to dataintensive fields such as bioinformatics, health care, and elderly care services. From the perspective of value dimensions, big data is a form of technology in potential resources.

It can be seen from the development of the economic field that big data has changed the economic and business models previously applied. Its application prospects in the social field are relatively broad, and it can also strengthen the social security level and the research and judgment of social policy trends. It is also under the influence of this trend that the implementation of data mining and statistical quantification based on the big data platform can determine the personalized and trend-oriented market demand and maintain the positioning of the law of development of things. It is also driven by big data that many changes in government functions such as models, services, and rules have also appeared, and the effects of upgrading and optimization are very obvious [1].

In general, elderly care services are not only a social issue, but also closely related to economic development. Moreover, the industry will be subject to many restrictions on its development. Through the application of emerging technologies such as big data and the Internet of Things, the quality and efficiency of elderly care services can be improved. This is also one of the effective measures to solve the pension problem. At the same time, the large number of households will create more conditions for smart, healthy and elderly care and create a new direction for digital development. Relevant departments need to provide intelligent and intelligent dynamic services for the elderly based on big data platforms and related technology products as carriers. This allows more people to enjoy the convenience brought by modern technology. 


\section{Modular Content of Elderly Care Services}

In the design of the "triad" model of elderly care services, the actual integration mechanism is mainly to couple and embed the elderly care resources of the family, community and institution. This model is also a representative of "Home Care Supported by Social Services and Community Services". At this stage, with the improvement of residents' living standards, the goals of elderly care services have shown a diversified development trend. It is precisely because of the improvement in the quality of elderly care services that the cost of elderly care services has also increased. The existence of the above-mentioned problems makes the "three-in-one" pension service model unable to truly penetrate the elderly. To this end, the relevant departments have implemented a modular processing analysis of the elderly care services, and the elderly can choose one or more of the services according to their specific needs. This can control the cost of elderly care and at the same time expand the audience for elderly care and establish a new benign ecosystem of elderly care services.

\subsection{Modular Design Ideas of Elderly Care Services}

In the actual application of elderly care service data, a corresponding mechanism should be established to divide elderly care services into independent service functional components according to the modularization concept. The achievement of elderly care services can be achieved by freely combining one or more service modules to establish new goals for elderly care services. The ideological design of the elderly service module can not only save a part of manpower and time, but also can customize a targeted service system according to the specific needs of the elderly. This will ensure the overall improvement of the quality of elderly care services and give the main body services more flexible characteristics. It can control service risks with the help of intensive and large-scale operations [2].

\subsection{Independence and Coupling Design of Elderly Care Service Modules}

The formulation of the actual personalized elderly care service system requires a combination of standardized modular design and intelligent combination methods. In the face of complex needs for elderly care services, we can start from the two characteristics of function and structure to realize a comprehensive analysis of elderly care service items. For those complex service goals, we can divide the service into common standard service units, so that each standard service unit can reach a unified pension goal. When different service units are combined, complex pension goals can be achieved. The modular application of elderly care services can strengthen the flexibility of elderly care services and meet the diverse needs of elderly care in the elderly care market. If the various standardized elderly service units are independent of each other, the units will not influence each other due to overlap and create favorable conditions for the recombination of subsequent service units. In contrast, the old-age service unit itself has obvious independence characteristics, and it can achieve comprehensive R\&D and innovation within the unit. At this time, we can use the information transmission of specific conditions and rules of the elderly service unit to create more complex elderly service goals. It is also in the design of this division of elderly care services that the service experience of the elderly can be substantially optimized. In turn, the service can be replicated and transplanted, showing better cycle characteristics.

\subsection{Modular Design Process for Elderly Care Services}

In view of the specific needs of the elderly, the staff needs to analyze the specific needs and simulate the entire elderly care service process. In addition, the staff must sort out the specific delivery process of elderly care services, clarify the content of new elderly care services, and construct elements of elderly care services. Only in this way can we provide effective hardware resources and equipment and service technical support for the main elderly care services. Then establish an elderly care service unit based on functional domains to ensure the comprehensiveness of elderly care services [3].

\section{Data Association Analysis of Distributed Elderly Care Services in Big Data Environment}

In recent years, big data technology has been widely used in the field of pension services, and governments at all levels have also strengthened the supply of big data and pension policies. We can analyze the big data generated by the big data pension service cloud platform design. This is also the process of further association and classification of elderly service units. In addition, we can construct specific theoretical models based on specific parameters of satisfaction with elderly care services to keep products synchronized with specific types of elderly care services. Only in this way can the goals of elderly care services and elderly care services be in a state of adaptation.

\subsection{Building A Distributed Associative Classifier}

The degree of achievement and satisfaction presented by the "three-in-one" elderly care service will be affected by many factors such as the health status of the elderly themselves, education level, etc., and also related to the specific situation and adaptation degree of the elderly care service provider. Relevant staff can use data mining association classification methods to determine a more suitable combination of elderly care service units, thereby creating favorable conditions for the subsequent modular implementation of elderly care services. Besides, the data warehouses used for service classification in the big data environment are distributed in different spaces, and 
different local elderly care information data sources can present different characteristics. At this point, in the specific classification process, people should establish a corresponding classifier according to the local data source situation and fully consider the characteristics of the local data source. It can be integrated with the classification results of other data sources, and horizontal weighting is used to compensate for the performance defects of the pension data set, so that the category distribution is in a balanced state. The specific measurement framework is the achievement and satisfaction degree of elderly care services, the classification of elderly care service units, and the creation of more new forms of elderly care systems [4].

\subsection{Incremental Mining of Association Classifiers Based on Constraints}

In the actual elderly care service classification application, the data used to classify elderly care data sets are often derived from continuously expanding incremental data. At this time, the classifier needs to make corresponding classification operations while adapting to the incremental data in a good state. In order to meet the needs of complex and diversified elderly care services, relevant departments should add constraints to the classifier and dynamically adjust the classification calculation method based on feedback from experts in the elderly care field. In this way, the class ensures that the classifier can quickly adapt to the incremental data and strengthen the accuracy of the classification of elderly care services. The actual association classifier mining process is shown in Figure 1.

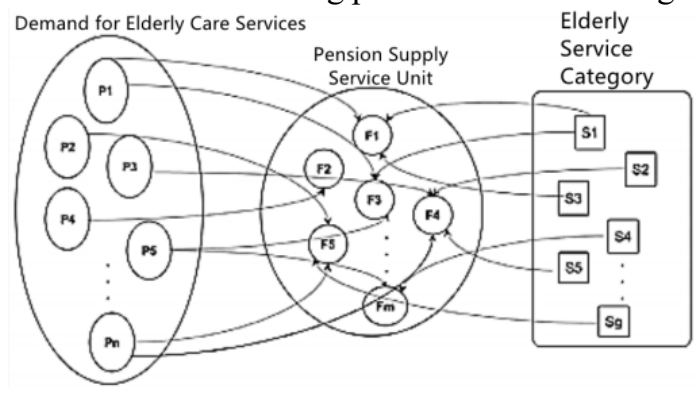

Fig1. Distributed Associative Classifier Mapping Process

\subsection{The Running Process of the Distributed Association Classifier Model}

Looking at the actual elderly care service data, the main source of data is the smart elderly care cloud platform. However, in the process of using these data, there may be incomplete or missing problems, and data redundancy is also accompanied. For this reason, before the execution of data mining operations, relevant staff need to clean up the basic data of elderly care services. In order to better implement the auxiliary classifier mining operation, the manager also needs to perform data conversion operations to keep the entire distributed association operation process in a reasonable state. In this way, you can enjoy the advantages of big data information sharing and ensure that the supply of elderly care services and the demand for elderly care services are in a state of matching with each other.

\subsection{Evaluation of Actual Elderly Care Services}

In the big data environment, we can gather information from multiple parties to establish a smart pension-related evaluation system with the help of big data technology. In this way, it is possible to adhere to the elderly as the center, realize the expansion of the index extraction range of the elderly care service evaluation system, and clarify the service data of the smart elderly cloud platform with the help of analysis and processing operations. The application of various evaluation algorithms and data mining can ensure accurate prediction of the effect of elderly care services, and we can improve and integrate the elderly care service process according to the actual situation and requirements. To achieve the above operations, the relevant staff must do the following work. Firstly, we should refine the observation points of various indicators in the evaluation of elderly care services. Secondly, we can use big data technology to realize the comprehensive processing of observing data of elderly care services. Finally, we can display the evaluation results to achieve standardized management of data. Otherwise, the staff will also feed back the evaluation results to the service classification and division module, establish a closed-loop pension service cycle model, and build a new pension service ecology. We can provide operational basis for follow-up quality evaluation and construction operations based on the "trinity" model.

\section{Current Status and Problems of China's Overall Elderly Care Services}

With the support of the Chinese government and relevant departments, the foundation of elderly care services has improved a lot, but there are still many problems. The collection of relevant data for the elderly files is too single, and the traditional electronic platform information storage cannot meet the current needs of elderly care services. Although this information involves many aspects of the elderly, such as physical status, children's conditions, etc., these data are difficult to be used effectively. Because these are basically invalid data, it is difficult to update the application in the future, and it is impossible to provide complete elderly care services for the elderly. More importantly, the information between the elderly on the demand side and the service supply side is asymmetrical and does not have the usual communication channels. Secondly, some social forces have not penetrated into the elderly care services. The core of China's elderly care services is mainly focused on enabling the elderly to enjoy personalized elderly care services related to all aspects of life without leaving home. On the demand side, the needs of the elderly are very diverse and trivial, and some important demand services are also involved. Up to now, these service agencies have not received specific social service forces and motivation support, which has greatly affected the elderly care services. In recent years, China's pension policy has gradually relaxed the requirements for the participation of social forces, which has also increased their enthusiasm for participation. However, the biggest difficulty currently facing is the inability to effectively 
integrate the human and material resources needed in social resources, which affects the effect of the entire elderly care service [5].

\section{The Development of my country's Elderly Service under the Big Data Environment}

\subsection{Pension Industry}

It can be seen from the actual development that big data based on human subjectivity has an increasingly obvious impact on the entire elderly care service industry. Through the mining of elderly care data, researchers can create new service content while discovering actual needs, thereby customizing new services and products. This process will also have an impact on data from manufacturing and financial industries, maintain the integrity of its own industrial structure, and ultimately establish a new industry chain for elderly care services. What'smore, many insurance companies themselves are not willing to provide related services for the elderly. This is mainly due to the unpredictable health status of the elderly themselves. At this time, the big data platform can provide help for the elderly, completely solve their worries, and then formulate insurance content that matches the elderly.

\subsection{Pension Service Guarantee}

Under the influence of the big data environment, China's elderly care services have entered a process of individualization and diversification. Moreover, we can make a comprehensive prediction of the follow-up pension trend based on data simulation technology, so that the backend governance will change to the front-end governance. In addition, we can also use intelligent learning technology as a basis to provide intelligent support for solving some major elderly care problems. This can reduce service costs while strengthening work effectiveness, and establish a warm and responsive elderly care service system. Relevant data show that as of 2018 , the scale of China's pension industry market has reached 6.57 trillion yuan, and it is expected that by 2021, this value will become 8.81 trillion yuan. At this stage, China's elderly care industry has formed specific suppliers of raw materials and energy, supply chains for supplies, and so on. In the first half of 2018, revenue exceeded trillions. In the future pension service security, China's pension services are mainly based on homes, supported by communities, and supplemented by institutions, which will promote the improvement of the entire pension service security market. It can also be seen from the market size that by 2022, the market size of China's pension industry will exceed 10 trillion yuan, and in 2018 , the value will be 6.57 trillion yuan. In contrast to the design of elderly care service institutions, the total number in China in 2017 was 155,000, a year-on-year increase of $10.7 \%$, and the number of newly registered elderly care service institutions was 29,000 . It is precisely because of the increase in the number of institutions that the number of beds has also shown an upward trend, but the growth rate is declining year by year. However, with the improvement of infrastructure construction, the supply of old-age beds will also return to the original [6].

\subsection{Government Governance}

In the big data environment, the existing behavior of the government itself will also change. For example, the application of big data can effectively break down barriers in civil affairs departments and eliminate all kinds of information islands. It can use information sharing and work linkage to ensure the smooth development of related work. In the establishment of actual elderly care services, the phenomenon of data disclosure will also be in a normalized state of development, and the effect of the public's supervision of the quality and content of service provision will also be revealed. Meanwhile, big data can enable the government to be more forward-looking in making decisions for the elderly, which is also an essential part of the construction of smart cities. In order to ensure the professionalization and systematization of elderly care services, relevant state departments have also issued many policies to point out the direction for the subsequent development of the elderly care service industry. For example, China advocates prioritizing the development of home network information services, and supporting corporate structures to rely on technologies such as big data This can ensure that the home care service model and content are more innovative. This is conducive to building a corresponding information open platform to provide consumers with comprehensive services.

\section{Conclusion}

In summary, the modularized treatment of elderly care services through the "three in one" can provide a new direction for the development of China's overall elderly care service industry. In addition, we can formulate personalized elderly care services according to specific needs, while providing intensive and large-scale services for elderly care providers to strengthen service quality. Furthermore, we need to use big data technology to achieve a comprehensive evaluation of the quality of elderly care services, channel more resources, and ensure that smart elderly care services are in a virtuous circle.

\section{Acknowledgment}

Funded project: Scientific research projects in higher education institutions of Inner Mongolia (NJSY18029)

\section{References}

1. Dong Xuan. Construction of the logistics supply chain of elderly service products under the background of the Internet $[\mathrm{J}]$. Logistics Technology, 2020, 39(11): 107-110+160.

2. Xiao Ping. How do social workers participate in the construction of the elderly service system[J]. China Social Work, 2020(33):1. 
3. Li Jintao, Chen Simin. Foreign public housing pension policies and community pension service allocation[J]. China Real Estate, 2020(33): 71-79.

4. Huang Lili, Wang Junjie. A qualitative study on the experience of nursing service quality management for managers in integrated medical and elderly care institutions[J]. Nursing and Rehabilitation, 2020, 19(11): 1-4+9.

5. Dang Liying, Gao Guiru. Feasibility Analysis of Rural Mutual Aid Pension Based on Time Bank Model[J]. Rural Economy and Technology, 2020, 31(21): 252-253.

6. Li Juan, Zhou Huaqing. Research on Hebei Province's Public Cultural Service System Construction from the Perspective of Cultural Aged Care[J]. Journal of North China University of Technology (Social Science Edition), 2020, 20(06): 53-58. 